\title{
Association between egg consumption and cardiovascular disease events, diabetes and all-cause mortality
}

\author{
Jing Guo ${ }^{1,3} \cdot$ Ditte A. Hobbs ${ }^{2,3} \cdot$ John R. Cockcroft ${ }^{4} \cdot$ Peter C. Elwood $^{5}$. \\ Janet E. Pickering ${ }^{1}$ Julie A. Lovegrove ${ }^{1,2,3} \cdot$ David I. Givens ${ }^{1,3}$
}

Received: 21 July 2017 / Accepted: 13 October 2017 / Published online: 2 November 2017

(c) The Author(s) 2017. This article is an open access publication

\begin{abstract}
Purpose The association between egg consumption and cardiovascular disease (CVD) or type 2 diabetes (T2D) remains controversial. We investigated the association between egg consumption and risk of CVD (primary outcome), T2D and mortality in the Caerphilly prospective cohort study (CAPS) and National Diet and Nutritional Survey (NDNS).

Methods CAPS included 2512 men aged 45-59 years (1979-1983). Dietary intake, disease incidence and mortality were updated at 5-year intervals. NDNS included 754 adults aged 19-64 years from 2008 to 2012.

Results Men free of CVD $(n=1781)$ were followed up for a mean of 22.8 years, egg consumption was not associated with new incidence of CVD $(n=715)$, mortality $(n=1028)$ or T2D $(n=120)$. When stroke $(n=248)$, MI $(n=477)$,
\end{abstract}

Supported by the Barham Benevolent Foundation.

Electronic supplementary material The online version of this article (doi:10.1007/s00394-017-1566-0) contains supplementary material, which is available to authorized users.

Julie A. Lovegrove

j.a.lovegrove@ reading.ac.uk

1 Institute for Food, Nutrition and Health, University of Reading, Reading RG6 6AR, UK

2 Hugh Sinclair Unit of Human Nutrition, University of Reading, Reading RG6 6AR, UK

3 Institute for Cardiovascular and Metabolic Research, University of Reading, Reading RG6 6AR, UK

4 Wales Heart Research Institute, Cardiff University, Cardiff, UK

5 Department of Primary Care and Public Health, Cardiff University, Cardiff, UK heart failure $(n=201)$ were investigated separately, no associations between egg consumption and stroke and MI were identified, however, increased risk of stroke in subjects with T2D and/or impaired glucose tolerance (IGT, fasting plasma glucose $\geq 6.1 \mathrm{mmol} / \mathrm{L}$ ), adjusted hazard ratios (95\% CI) were 1.0 (reference), 1.09 (0.41, 2.88), $0.96(0.37,2.50)$, $1.39(0.54,3.56)$ and $2.87(1.13,7.27)$ for egg intake $(n)$ of $0 \leq n \leq 1,1<n \leq 2,2<n \leq 3,3<n<5$, and $n \geq 5$ eggs/wk, respectively $(P=0.01)$. In addition, cross-sectional analyses revealed that higher egg consumption was significantly associated with elevated fasting glucose in those with T2D and/or IGT (CAPS: baseline $P=0.02$ and 5-year $P=0.04$; NDNS: $P=0.05$ ).

Conclusions Higher egg consumption was associated with higher blood glucose in subjects with T2D and/or IGT. The increased incidence of stroke with higher egg consumption among T2D and/or IGT sub-group warrants further investigation.

Keywords Eggs $~$ Cardiovascular disease $\cdot$ Type 2 diabetes $\cdot$ Impaired glucose tolerance $\cdot$ All-cause mortality

$\begin{array}{ll}\text { Abbreviations } \\ \text { CVD } & \text { Cardiovascular disease } \\ \text { MI } & \text { Myocardial infarction } \\ \text { T2D } & \text { Type 2 diabetes } \\ \text { IGT } & \text { Impaired glucose tolerance } \\ \text { CAPS } & \text { Caerphilly prospective cohort study } \\ \text { NDNS } & \text { National diet and nutrition survey } \\ \text { FFQ } & \text { Food-frequency questionnaire } \\ \text { RCT } & \text { Randomized controlled trials } \\ \text { SBP } & \text { Systolic blood pressure } \\ \text { DBP } & \text { Diastolic blood pressure } \\ \text { TAG } & \text { Triacylglycerol } \\ \text { CRP } & \text { C-reactive protein }\end{array}$


HbA1c Glycated haemoglobin

ICD International Classification of Diseases

HR Hazard ratio

\section{Introduction}

Cardiovascular diseases (CVD) are still the leading cause of morbidity and mortality, and the prevalence of type 2 diabetes mellitus (T2D) is also increasing globally. Diet plays an important role in prevention and management of both CVD [1] and T2D [2]. Eggs are a good source of a number of nutrients in the UK diet such as vitamin $\mathrm{D}$, selenium, vitamin $\mathrm{K}$ and choline as well as high quality protein [3]. However, eggs also contain relatively high amounts of dietary cholesterol $(350 \mathrm{mg} / 100 \mathrm{~g})$ [4], which has been associated with impaired glucose metabolism [5] and increased inflammation [6] in animal models and with elevated fasting glucose in humans [7]. Furthermore, meta-analyses of intervention studies have shown that high consumption of dietary cholesterol increases serum total, LDL- and HDL-cholesterol concentrations, as well as the ratio of total to HDLcholesterol $[8,9]$, all key risk factors of CVD, although it has been proposed that these changes are small and are not clinically significant [10].

Evidence for the association between egg consumption and glycaemic control is limited. Findings from a randomized controlled trial (RCT) of 140 overweight or obese subjects with prediabetes or T2D showed that high-egg consumption ( 2 eggs/day for 6 days/week) did not adversely effect lipid profiles of those with T2D [11]. Furthermore, Fuller et al. [11] reported no effect of egg intake on glycaemic control in a 3-month RCT in subjects with T2D, whereas an inverse association between egg intake and fasting plasma glucose was reported in a prospective cohort study [12]. Moreover, evidence from previous meta-analyses in relation to egg consumption and CVD risk showed inconsistent results. Some studies $[13,14]$ have shown that consumption of up to one egg per day is not associated with increased risk of CVD in the general population, which is in contrast to a recent meta-analysis which reported up to one egg per day was associated with reduced risk of stroke [15]. Furthermore, inconsistent associations between egg intake and CVD in diabetic patients were observed. Shin et al. [14] concluded that consumption of up to one egg per day was associated with an increased risk of CVD in those suffering from T2D, whereas Rong et al. [13] found intake of up to one egg per day was associated with an increased risk of CHD in diabetic patients, but may have contributed to a reduced risk of hemorrhagic stroke in this sub-group.

Due to the inconsistent reported relationship between egg consumption and cardio-metabolic disease incidence and risk we investigated the association between egg consumption and incidence of CVD (primary outcome) and allcause mortality and incidence of T2D (secondary outcomes) in middle-aged men from the UK Caerphilly Prospective Cohort Study (CAPS). Furthermore, due to the different pathophysiologies of diseases classed as CVD, including myocardial infarction (MI), stroke and heart failure [16], the relationship between these diseases and egg consumption were further investigated separately. Furthermore, the cross-sectional relationships between egg intake and a number of cardiometabolic risk markers were investigated using data from the CAPS and the UK National Diet and Nutrition Survey (NDNS) rolling programme (years 1-4, secondary outcomes) cross-sectionally. Our hypothesis was that a higher egg intake was unlikely to increase the risk of CVD (including MI, stroke, heart failure and cardio-metabolic risk markers), T2D or all-cause mortality, but may have detrimental effects in those suffering from T2D.

\section{Subjects and methods}

\section{Caerphilly prospective cohort study}

\section{Study population of caerphilly prospective cohort study}

The CAPS was set up between 1979 and 1983 and was designed to investigate CVD risk factors in men, there was follow-up of the men who were re-examined at 5-year intervals (Fig. 1). Initially, 2512 men aged 45-59-years-old living in Caerphilly, Wales, United Kingdom and the adjacent area were recruited onto the study [17]. However, 561 men decided to leave the study after Phase 1 (1979-1983) and an additional group of 447 men were recruited for replacement, giving a new total of 2398 men at Phase 2 (1984-1988). At 5 years later, a total of 2147 men revisited the clinical centre for the Phase 3 examination (1989-1993). The current study has not included data from Phase 1 , as there were an inconsistent population and number of subjects between Phase 1 and Phase 2.
Fig. 1 Timeline of the caerphilly prospective cohort study

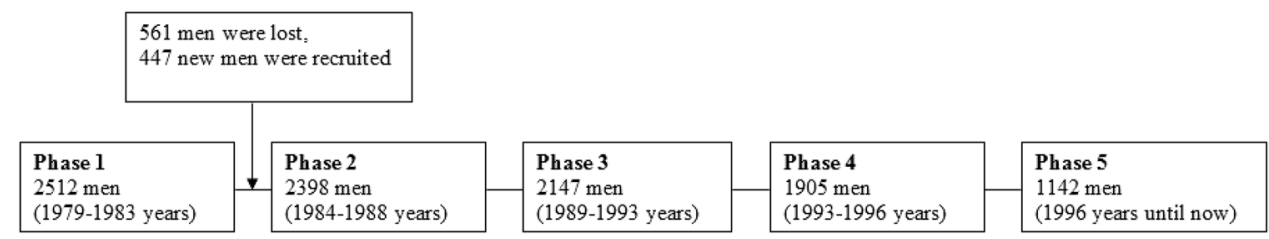




\section{Dietary assessment}

Diet was assessed at both Phase 2 and Phase 3 with the use of a semi-quantitative food-frequency questionnaire (FFQ). Participants were asked to report the number of eggs consumed on a weekly basis, with one unit of consumption equivalent to one egg, however, this excluded eggs present in composite dishes. This FFQ was previously validated using a 7-day weighed diet diary in a representative subgroup of 665 men, representing $30 \%$ of Phase 1 participants [18]. To present the best estimation of egg consumption, the mean egg intake of Phase 2 and Phase 3 was used for the longitudinal analysis, which also allowed estimation of the effect of the cumulative long-term diet. Subjects with preexisting stroke $(n=60)$, MI $(n=98)$, and those with missing data (including subjects who dropped out during the followup period) or confounding factors $(n=208)$ were excluded from the longitudinal CVD analysis, which left a total of 1781 men. Subjects with pre-existing stroke, MI were also excluded from cross-sectional analyses at Phase 2 or Phase 3. Furthermore, subjects with pre-existing T2D $(n=94)$ were excluded from the longitudinal T2D analysis, which left a total 1687 men. The mean egg intakes at Phase 2 and Phase 3 were calculated for all of the subjects who reported egg consumption in both phases. Egg consumption ( $n=$ number/ week) was divided into five groups for the analysis: $0 \leq n \leq 1$, $1<n \leq 2,2<n \leq 3,3<n<5, n \geq 5$.

\section{Cardiovascular events, diabetes and all-cause mortality}

The incidence of T2D was self-reported from questionnaires in the CAPS. Identification of vascular disease events and deaths by cause has been described elsewhere [19-21]. In brief, subjects were seen in a central clinic, symptoms and illnesses suggestive of a stroke or heart attack were confirmed by the use of the London School of Hygiene chest pain questionnaire and the Oxford Stroke Questionnaire; subjects also had an electrocardiogram measurement during the visit. Appropriate searches of hospital and general practitioner databases were made to extract relevant clinical information. Vascular events (International Classification of Diseases (ICD) 121-5, 10th revision) of fatal ischaemic heart disease and non-fatal MI and ischaemic stroke (ICD 163-4) were diagnosed by two independent expert clinicians and an epidemiologist using all available clinical evidence, including computed tomography, radiological and pathological information. Furthermore, the records of all men in the National Health Service Central Registry were flagged so that notification of death certificate was received automatically, and cause of death was defined by ICD-9 Revision.

The aim of the present study was to investigate the relationship between egg intake and CVD or mortality in the total population, as well as in a sub-group suffering from
T2D and/or impaired glucose tolerance (IGT). After removal of 16 subjects with T2D who had missing dietary or confounding factor data from baseline, 94 pre-existing T2D subjects remained for inclusion in the analysis. To have sufficient numbers for the statistical analysis, we combined the men with IGT $(n=319)$, defined by the WHO as fasting plasma glucose of $6.1 \mathrm{mmol} / \mathrm{L}$ or higher [22]. Subjects $(n=73)$ who met the inclusion criteria for both T2D and IGT were counted once in the analysis, thus, T2D and/or IGT sub-group included 340 subjects which were included in the longitudinal analysis of the associations between egg consumption with CVD events or all-cause mortality.

\section{Other measurements}

Systolic blood pressure (SBP), diastolic blood pressure (DBP), plasma glucose, insulin, triacylglycerol (TAG), total/ HDL-cholesterol, fibrinogen, homocysteine and C-reactive protein (CRP) were measured in fasting plasma or serum samples at Phase 2. LDL-cholesterol was calculated using TAG, total cholesterol and HDL-cholesterol by the Friedewald formula [23]. Pulse pressure was calculated by subtracting DBP from SBP. However, only SBP, DPB, glucose, TAG, total cholesterol, fibrinogen were measured at Phase 3 in CAPS.

\section{National diet and nutrition survey}

Data files from the National Diet and Nutrition Survey (NDNS) [24] years 1-4 of the rolling programme (2008-2009 to 2011-2012) were obtained from the UK Data Archive (http://www.data-archive.ac.uk). The data from 754 adults (males $n=322$ and females $n=432$ ) aged 19-64 years were used to determine association between egg intake (g/ day) and fasting blood glucose, glycated haemoglobin (HbA1c) and other biochemical measures of cardio-metabolic health, including total-, HDL-, and LDL- cholesterol, the total-/HDL- cholesterol ratio, TAG, SBP, DBP, pulse pressure and CRP. Food intake was collected using 4-day food dairy. Participants with a previous history of stroke $(n=1)$, heart attack or angina $(n=6)$ were excluded from the analysis. In addition, associations between egg consumption and metabolic markers were examined in a T2D and/or IGT sub-group, which included subjects with T2D $(n=14)$ or IGT (fasting plasma glucose $\geq 6.1 \mathrm{mmol} / \mathrm{L}, n=56$ ), subjects $(n=11)$ who met inclusion criteria for both T2D and IGT groups were only counted once. Thus, the total number in the T2D and/or IGT sub-group was 59 subjects. The egg food group included whole eggs and dishes such as omelettes and scrambled eggs, which was analysed as continuous variable (with increments of $100 \mathrm{~g} / \mathrm{day}$ ). Composite dishes such as egg fried rice and quiches were removed from the food group to fit with the analysis conducted on the CAPS. 


\section{Statistical analysis}

\section{Longitudinal investigation in CAPS}

All data analysis was conducted using STATA (version 13.0; STATA Corporation, 2014) and a 2-sided $p<0.05$ was considered statistically significant. In the longitudinal analysis of CAPS, Cox proportional hazard models were used to calculate non-adjusted and multivariate adjusted hazard ratios (HR) by comparing the time until onset of disease or mortality in subjects in the highest intake categories of egg consumption with that in the lowest egg group as the reference group. The survival time for each endpoint (MI, stroke, heart failure, T2D or mortality) was the first occurrence of an endpoint, death, or the date of receipt of the last followup questionnaire. If subjects had a non-fatal event these data were used rather than the later date of death.

The first multivariate model controlled for a number of confounding factors in CAPS. These included the covariates age (years), body mass index (weight $(\mathrm{kg}) /$ height $\left(\mathrm{m}^{2}\right)$ ), energy intake (kJ/day), alcohol consumption (as ethanol, $\mathrm{ml}$ /week), smoking (never-smoked, ex-smoked, current smoked), energy expenditure ( $\mathrm{kJ} /$ day), social class (manual worker; non-manual worker), family history of MI (yes or no) and T2D (yes or no). The second multivariate model also controlled for sugar intake $(<50,50-100,>100 \mathrm{~g} /$ day $)$, fruit consumption $(<7,8-14,15-21$, or $>21$ times/week), red meat consumption $(<7,8-14,15-21$, or $>21$ times/week) and fibre intake (cereal and vegetable sources) $(<10,10-20$, or $>20 \mathrm{~g} / \mathrm{day}$ ). The possibility of an interaction between egg consumption and subgroup of T2D and/or IGT with respect to any of the outcomes was investigated by an analysis including an interaction term in the Cox proportional hazard models.

\section{Cross-sectional analysis in CAPS and NDNS}

In secondary analyses, we examined the association between egg consumption and metabolic markers in CAPs and NDNS. For cross-sectional analysis of CAPs, the association of egg consumption with a range of metabolic markers was examined at Phase 2 . In the sensitivity analyses of crosssectional analysis, the associations of egg consumption with metabolic markers were also evaluated at Phase 3 to test the consistency of the findings. Trends associated with increasing egg consumption were investigated using linear regression for the continuous variables and logistic regression for categorical variables. The same confounding factors used for the multivariate models of the longitudinal analysis in CAPS were applied in the cross-sectional analysis.

For cross-sectional analysis of the NDNS, the confounding factors used were age (years), gender (men or women), energy intake (kJ/day), alcohol consumption (as ethanol, g/ day), T2D (yes or no) and smoking habit (smokers or nonsmokers). General linear regression was used for the analysis. Original data were transformed to natural logarithms if required.

\section{Results}

\section{CAPS: baseline characteristics according to egg consumption}

The mean egg intake of 1781 subjects was $2.9(\mathrm{SD}=2.1)$ eggs per week, and ranged from 0 to 34 eggs per week. Among participants, $14.4 \%$ subjects consumed 5 eggs or more per week. The baseline characteristics of the subjects from the CAPS are shown in Table 1. The men in the highest quantiles of egg consumption were significantly more likely to be manual workers, smokers, consume more alcohol, have a higher energy intake and expenditure and a higher BMI. They also had a lower incidence of family MI history. After controlling for energy intake from foods, the men with the highest egg consumption had a significantly higher intake of total fat, saturated fat and sugar, but lower cereal or vegetable fibre, carbohydrate intakes, red meat and fruit intake.

\section{CAPS: egg consumption and CVD events, all-cause mortality and diabetes in longitudinal investigation}

During the mean follow-up of 22.8 years, incident cases of total CVD event $(n=715)$ and all-cause mortality $(n=1028)$ were reported in the subjects initially free from CVD events (Table 2). When different types of CVD event were investigated separately, egg consumption was not associated with incidence of MI $(n=477)$ or heart failure $(n=201)$. However, a significant trend of higher risk of stroke with increasing egg intake (adjusted model $P=0.04$ ) was observed, with HR of 1.60 (95\% CI: 1.00, 2.57) for the highest $(\geq 5$ eggs/week) vs lowest $(0 \leq$ eggs/week $\leq 1)$ quantile of egg consumption.

In stratified analyses, the prevalence of T2D and/or IGT did not influence the association between egg consumption and total CVD, MI, heart failure, or all-cause mortality (data not shown). When the subjects with T2D and/or IGT were removed from the analysis of stroke, there was no significant increase in risk of stroke across increasing quantiles of egg consumption (Table 3). However, when this analysis was performed on the T2D and/or IGT sub-group, a significant trend for increased risk of stroke (adjusted model $P=0.01)$ with increasing egg consumption was identified, HR for incident stroke was $2.87(95 \%$ CI 1.13, 7.27) in the highest vs lowest quantile of egg consumption ( $P$ for interaction between egg consumption and T2D and/or IGT $=0.09$ 
Table 1 Baseline characteristics of participants from the caerphilly prospective cohort study according to egg consumption

\begin{tabular}{|c|c|c|c|c|c|c|}
\hline \multirow[t]{2}{*}{ Characteristics } & \multicolumn{5}{|c|}{ Egg consumption ( $n$, eggs/wk) } & \multirow[t]{2}{*}{$P$ trend $^{\mathrm{a}}$} \\
\hline & $(0 \leq \mathrm{n} \leq 1)$ & $(1<\mathrm{n} \leq 2)$ & $(2<\mathrm{n} \leq 3)$ & $(3<\mathrm{n}<5)$ & $(\mathrm{n} \geq 5)$ & \\
\hline Total subjects, $n$ & 274 & 464 & 469 & 318 & 256 & \\
\hline Age, years & $61.5 \pm 4.6$ & $61.9 \pm 4.3$ & $61.7 \pm 4.5$ & $61.7 \pm 4.5$ & $61.6 \pm 4.4$ & 0.93 \\
\hline BMI, $\mathrm{kg} / \mathrm{m}$ & $26.5 \pm 3.5$ & $26.5 \pm 3.4$ & $26.7 \pm 3.7$ & $26.9 \pm 4.0$ & $27.1 \pm 4.2$ & 0.03 \\
\hline Energy expenditure, $\mathrm{kJ} /$ day & $1440 \pm 1557$ & $1421 \pm 1426$ & $1551 \pm 1651$ & $1659 \pm 1618$ & $1850 \pm 2042$ & 0.001 \\
\hline Manual workers, $\%$ & 53.3 & 59.7 & 63.5 & 70.8 & 80.1 & $<0.001$ \\
\hline Family history of MI, $\%$ & 43.2 & 38.6 & 38.4 & 30.4 & 36.7 & 0.02 \\
\hline History of hypertension, $\%$ & 28.8 & 26.9 & 29.2 & 31.1 & 29.7 & 0.40 \\
\hline History of diabetes, $\%$ & 3.3 & 1.7 & 0.9 & 5.3 & 3.9 & 0.07 \\
\hline Current smokers, $\%$ & 22.6 & 26.9 & 36.2 & 43.4 & 48.0 & $<0.001$ \\
\hline Total energy intake, $\mathrm{kJ} / \mathrm{day}$ & $7449 \pm 1890$ & $8074 \pm 1873$ & $8547 \pm 2051$ & $8988 \pm 2132$ & $9821 \pm 2578$ & $<0.001$ \\
\hline Fat, $\%$ of total energy & 33.9 & 35.1 & 35.8 & 36.5 & 37.1 & $<0.001$ \\
\hline Saturated fat, $\%$ of total energy & 14.6 & 15.0 & 15.5 & 15.9 & 16.1 & $<0.001$ \\
\hline Protein, $\%$ of total energy & 14.7 & 14.3 & 14.3 & 14.2 & 14.5 & 0.19 \\
\hline Carbohydrates, $\%$ of total energy & 49.3 & 48.3 & 47.6 & 47.1 & 45.9 & $<0.001$ \\
\hline Fibre (vegetable sources) ${ }^{\mathrm{b}}, \mathrm{g} /$ day & $11.4 \pm 0.5$ & $10.9 \pm 0.5$ & $11.1 \pm 0.6$ & $11.2 \pm 0.6$ & $11.3 \pm 0.7$ & 0.02 \\
\hline Fibre (cereal sources) ${ }^{\mathrm{b}}, \mathrm{g} / \mathrm{day}$ & $10.8 \pm 1.2$ & $10.3 \pm 1.1$ & $10.0 \pm 1.3$ & $9.3 \pm 1.3$ & $9.2 \pm 1.6$ & $<0.001$ \\
\hline Sugar ${ }^{\mathrm{b}}, \mathrm{g} /$ day & $76.8 \pm 24.1$ & $82.2 \pm 23.9$ & $87.3 \pm 26.1$ & $93.6 \pm 27.2$ & $104.3 \pm 32.9$ & $<0.001$ \\
\hline Fruit $^{\mathrm{b}}$, number/week & $9.3 \pm 0.5$ & $8.3 \pm 0.5$ & $7.9 \pm 0.6$ & $7.4 \pm 0.6$ & $7.7 \pm 0.7$ & $<0.001$ \\
\hline Vegetable $^{\mathrm{b}}$, times/week & $10.7 \pm 4.7$ & $10.0 \pm 4.6$ & $10.5 \pm 4.7$ & $10.6 \pm 4.8$ & $10.3 \pm 4.8$ & 0.99 \\
\hline Red meat ${ }^{\mathrm{b}}$, times/week & $17.4 \pm 0.9$ & $14.9 \pm 0.9$ & $14.8 \pm 1.0$ & $14.3 \pm 1.1$ & $14.8 \pm 1.3$ & $<0.001$ \\
\hline Ethanol intake, ml/week & $15.6 \pm 21.5$ & $16.2 \pm 20.3$ & $17.8 \pm 22.5$ & $17.3 \pm 19.7$ & $20.5 \pm 22.8$ & 0.010 \\
\hline
\end{tabular}

All values are mean $\pm \mathrm{SD}$

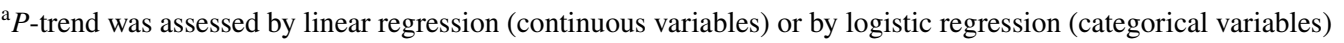

${ }^{\mathrm{b}}$ Energy-adjusted values

in non-adjust model, 0.08 in adjusted Model 1 and 0.07 in adjusted Model 2).

During the follow-up, a total of 120 new T2D cases were diagnosed for subjects initially free from CVD and T2D events. There was no association between egg consumption and incident T2D using either un-adjusted or multiple adjusted model (Table 4).

\section{CAPS and NDNS: associations between egg consumption and cardio-metabolic risk factors: cross-sectional analysis}

In Phase 2 of the CAPS, no associations were found between egg consumption and fasting plasma glucose concentration in the subjects free from CVD (Supplemental Table 1). However, when the analysis was repeated in a subgroup $(n=268)$ of participants with T2D and/or IGT, there was a significant positive association between egg consumption and fasting glucose concentration (Supplemental Table 2). In this subgroup analysis subjects consuming $\geq 5$ eggs/week had $1.31 \mathrm{mmol} / \mathrm{L}$ higher fasting glucose compared with subjects consuming 1 eggs/wk or less. There were no significant associations between egg consumption and other biomarkers of CVD risk in the population as a whole (Supplemental Table 1) and in subgroup analysis (Supplemental Table 2).

In Phase 3 of the CAPS there was a significant positive association between egg consumption and fasting glucose concentration (Supplemental Table 3). When the subjects with T2D and/or IGT were removed from the analysis, there was no association between egg consumption and fasting glucose concentration across increasing quantile of egg consumption. However, when the analysis was repeated in the subgroup of T2D and/or IGT $(n=334)$ there was a significant positive association between egg consumption and fasting glucose (Supplemental Table 4). In this subgroup analysis subjects consuming $\geq 5$ eggs per week had $0.72 \mathrm{mmol} / \mathrm{L}$ higher fasting glucose levels compared with subjects consuming $\leq 1$ eggs/week.

Cross-sectional analysis in NDNS [24] showed egg consumption was positively associated with $\mathrm{HbAlc}$ concentrations (Supplemental Table 5). When the subjects with T2D and/or IGT were removed from the analysis, there remained a significant positive trend for increased egg consumption and HbA1c concentration $(P=0.02)$. In the T2D and/or IGT subgroup, egg consumption was significantly associated with elevated fasting glucose and $\mathrm{HbA} 1 \mathrm{c}$ concentrations 
Table 2 Longitudinal study of incidence of stroke, myocardial infarction, heart failure and all-cause mortality according to egg consumption of all subjects from the caerphilly prospective cohort study

\begin{tabular}{|c|c|c|c|c|c|c|}
\hline \multirow[t]{2}{*}{ Characteristics } & \multicolumn{5}{|c|}{ Egg consumption ( $n$, eggs/week) } & \multirow[t]{2}{*}{$P$-trend } \\
\hline & $(0 \leq n \leq 1)$ & $(1<n \leq 2)$ & $(2<n \leq 3)$ & $(3<n<5)$ & $(n \geq 5)$ & \\
\hline Total subjects, $n$ & 274 & 464 & 469 & 318 & 256 & \\
\hline \multicolumn{7}{|l|}{ Total CVD events } \\
\hline No. of events & 103 & 166 & 195 & 129 & 122 & \\
\hline HR (non-adjust) & 1 & $0.95(0.74,1.21)$ & $1.12(0.88,1.42)$ & $1.08(0.83,1.40)$ & $1.37(1.05,1.78)$ & 0.007 \\
\hline HR (adjusted Model 1) ${ }^{\mathrm{a}}$ & 1 & $0.97(0.75,1.24)$ & $1.11(0.87,1.42)$ & $1.02(0.78,1.34)$ & $1.26(0.95,1.67)$ & 0.09 \\
\hline HR (adjusted Model 2) ${ }^{\mathrm{b}}$ & 1 & $0.98(0.76,1.26)$ & $1.14(0.89,1.46)$ & $1.01(0.77,1.33)$ & $1.25(0.94,1.66)$ & 0.12 \\
\hline \multicolumn{7}{|l|}{ Stroke } \\
\hline No. of events & 33 & 57 & 57 & 48 & 53 & \\
\hline HR (non-adjust) & 1 & $1.01(0.66,1.56)$ & $1.01(0.66,1.55)$ & $1.27(0.82,1.98)$ & $1.82(1.18,2.80)$ & 0.002 \\
\hline HR (adjusted Model 1) ${ }^{\mathrm{a}}$ & 1 & $0.99(0.65,1.53)$ & $0.97(0.63,1.50)$ & $1.14(0.72,1.81)$ & $1.58(1.00,2.52)$ & 0.03 \\
\hline HR (adjusted Model 2) & 1 & $1.01(0.65,1.56)$ & $1.00(0.64,1.55)$ & $1.15(0.72,1.84)$ & $1.60(1.00,2.57)$ & 0.04 \\
\hline \multicolumn{7}{|l|}{ Myocardial infarction } \\
\hline No. of events & 73 & 117 & 137 & 86 & 64 & \\
\hline HR (non-adjust) & 1 & $0.94(0.70,1.26)$ & $1.11(0.83,1.47)$ & $1.00(0.73,1.37)$ & $0.94(0.67,1.31)$ & 0.98 \\
\hline HR (adjusted Model 1) & 1 & $0.96(0.71,1.29)$ & $1.10(0.83,1.48)$ & $0.99(0.71,1.37)$ & $0.90(0.63,1.29)$ & 0.75 \\
\hline HR (adjusted Model 2) & 1 & $0.97(0.72,1.31)$ & $1.14(0.85,1.52)$ & $1.01(0.72,1.40)$ & $0.91(0.64,1.31)$ & 0.80 \\
\hline \multicolumn{7}{|l|}{ Heart failure } \\
\hline No. of events & 29 & 33 & 63 & 44 & 32 & \\
\hline HR (non-adjust) & 1 & $0.66(0.40,1.09)$ & $1.29(0.83,2.00)$ & $1.33(0.83,2.13)$ & $1.20(0.72,1.98)$ & 0.03 \\
\hline HR (adjusted Model 1) & 1 & $0.64(0.39,1.06)$ & $1.17(0.74,1.83)$ & $1.10(0.67,1.79)$ & $0.89(0.52,1.52)$ & 0.46 \\
\hline HR (adjusted Model 2) & 1 & $0.65(0.39,1.08)$ & $1.19(0.76,1.88)$ & $1.09(0.66,1.81)$ & $0.89(0.51,1.53)$ & 0.49 \\
\hline \multicolumn{7}{|l|}{ All-cause mortality } \\
\hline No. of events & 135 & 249 & 293 & 187 & 164 & \\
\hline HR (non-adjust) & 1 & $1.13(0.92,1.40)$ & $1.35(1.10,1.66)$ & $1.25(1.00,1.56)$ & $1.44(1.14,1.80)$ & 0.001 \\
\hline HR (adjusted Model 1) & 1 & $1.08(0.87,1.33)$ & $1.21(0.98,1.49)$ & $1.03(0.82,1.30)$ & $1.11(0.87,1.42)$ & 0.58 \\
\hline HR (adjusted Model 2) & 1 & $1.08(0.87,1.34)$ & $1.20(0.98,1.49)$ & $1.02(0.81,1.29)$ & $1.08(0.84,1.38)$ & 0.80 \\
\hline
\end{tabular}

${ }^{a}$ Values are hazard ratios (95\% CIs) derived by Cox proportional hazards regression models adjusted for age (continuous), BMI (continuous), total energy intake (continuous), alcohol consumption (quartiles), smoking (never, past or current), energy expenditure (quartiles), social class (manual or non-manual), family history of myocardial infarction (yes or no), diabetes mellitus (yes or no)

${ }^{\mathrm{b}}$ Adjusted as model 1 plus sugar intake $(<50,50-100,>100 \mathrm{~g} /$ day $)$, fruit consumption $(<7,8-14,15-21$, or $>21$ times/week), red meat consumption $(<7,8-14,15-21$, or $>21$ times/week) and fibre (cereal and vegetable sources) $(<10,10-20$, or $>20$ g/day)

( $P=0.05$; Supplemental Table 6). There were no significant associations between egg consumption and other markers of cardiovascular risk (Supplemental Table 5).

\section{Discussion}

In the CAPS no overall associations between weekly egg consumption up to 5 eggs/week and risk of total CVD, MI, heart failure, all-cause mortality and T2D were observed after a mean follow-up of 22.8 years, but there was a significant positive trend in stroke risk across the quantiles of egg intake. Further sub-group analysis showed that the significant trend disappeared after the men with T2D and/or IGT were removed. However the association of egg intake and risk of stroke in the sub-group of T2D and/or IGT only, showed significant positive associations. Secondary analyses in both CAPS and NDNS showed increased fasting glucose with higher egg intake in the sub-group of T2D and/ or IGT. In addition, results of cross-sectional analyses of NDNS showed higher egg intake was associated with higher $\mathrm{HbA} 1 \mathrm{c}$ in the general healthy population across tertiles of egg intake.

These results are consistent with the previous metaanalyses of prospective studies $[13,14]$, which showed no association between egg consumption and CVD events in the general population. Very few studies have reported a positive association between egg intake and CVD risk. Nevertheless, data from Physicians' Health Study [25] indicated the HR of heart failure was 1.33 (95\% CI 1.04, 1.70) with 
Table 3 Longitudinal analysis of incident of stroke according to egg consumption in subjects with and without type 2 diabetes and/or impaired glucose tolerance from the Caerphilly Prospective Cohort study

\begin{tabular}{|c|c|c|c|c|c|c|}
\hline & \multicolumn{5}{|c|}{ Egg consumption (n, eggs/week) } & \multirow[t]{2}{*}{$P$-trend } \\
\hline & $(0 \leq n \leq 1)$ & $(1<n \leq 2)$ & $(2<n \leq 3)$ & $(3<n<5)$ & $(n \geq 5)$ & \\
\hline \multicolumn{7}{|c|}{ Subjects without $\mathrm{T} 2 \mathrm{D}$ and/or IGT ${ }^{\mathrm{a}}$} \\
\hline Total subjects, $n$ & 221 & 397 & 378 & 248 & 197 & \\
\hline No. of events & 25 & 47 & 45 & 34 & 31 & \\
\hline HR (non-adjust) & 1 & $1.05(0.64,1.70)$ & $1.05(0.65,1.72)$ & $1.23(0.73,2.05)$ & $1.45(0.85,2.45)$ & 0.12 \\
\hline HR (adjusted Model 1) ${ }^{\mathrm{b}}$ & 1 & $1.01(0.62,1.66)$ & $0.97(0.59,1.60)$ & $1.14(0.67,1.95)$ & $1.28(0.73,2.24)$ & 0.33 \\
\hline HR (adjusted Model 2) ${ }^{\mathrm{c}}$ & 1 & $1.05(0.64,1.71)$ & $1.01(0.61,1.66)$ & $1.17(0.68,2.02)$ & $1.32(0.75,2.34)$ & 0.29 \\
\hline \multicolumn{7}{|c|}{ Subjects with T2D and/or IGT } \\
\hline Total Subjects, $\mathrm{n}$ & 53 & 67 & 91 & 70 & 59 & \\
\hline No. of events & 8 & 10 & 12 & 14 & 22 & \\
\hline HR (non-adjust) & 1 & $0.96(0.38,2.44)$ & $0.86(0.35,2.10)$ & $1.35(0.57,3.23)$ & $2.71(1.21,6.09)$ & 0.003 \\
\hline HR (adjusted Model 1) & 1 & $1.10(0.42,2.86)$ & $1.02(0.40,2.62)$ & $1.35(0.53,3.43)$ & $2.83(1.15,6.96)$ & 0.01 \\
\hline HR (adjusted Model 2) & 1 & $1.09(0.41,2.88)$ & $0.96(0.37,2.50)$ & $1.39(0.54,3.56)$ & $2.87(1.13,7.27)$ & 0.01 \\
\hline
\end{tabular}

${ }^{a}$ Impaired glucose tolerance, i.e., fasting glucose $\geq 6.1 \mathrm{mmol} / \mathrm{L}$

${ }^{b}$ Values are hazard ratios (95\% CIs) derived by Cox proportional hazards regression models adjusted for age (continuous), BMI (continuous), total energy intake (continuous), alcohol consumption (quartiles), smoking (never, past or current), energy expenditure (quartiles), social class (manual or non-manual), family history of myocardial infarction (yes or no)

${ }^{\mathrm{c}}$ Adjusted as model 1 plus sugar intake $(<50,50-100,>100 \mathrm{~g} /$ day $)$, fruit consumption $(<7,8-14,15-21$, or $>21$ times/week), red meat consumption $(<7,8-14,15-21$, or $>21$ times/week) and fibre (cereal and vegetable sources) $(<10,10-20$, or $>20$ g/day)

Table 4 Longitudinal study of incidence of type 2 diabetes according to egg consumption from the caerphilly prospective cohort study

\begin{tabular}{|c|c|c|c|c|c|c|}
\hline & \multicolumn{5}{|c|}{ Egg consumption ( $n$, eggs/week) } & \multirow[t]{2}{*}{$P$-trend } \\
\hline & $(0 \leq n \leq 1)$ & $(1<n \leq 2)$ & $(2<n \leq 3)$ & $(3<n<5)$ & $(n \geq 5)$ & \\
\hline Total subjects, $n$ & 259 & 447 & 453 & 290 & 238 & \\
\hline No. of events & 17 & 31 & 35 & 21 & 16 & \\
\hline HR (non-adjust) & 1 & $1.17(0.65,2.12)$ & $1.24(0.69,2.21)$ & $1.20(0.63,2.27)$ & $1.22(0.61,2.41)$ & 0.59 \\
\hline HR (adjusted Model 1) ${ }^{\mathrm{a}}$ & 1 & $1.08(0.59,1.97)$ & $1.05(0.57,1.92)$ & $1.25(0.64,2.44)$ & $1.23(0.60,2.53)$ & 0.48 \\
\hline HR (adjusted Model 2) ${ }^{\mathrm{b}}$ & 1 & $1.05(0.57,1.93)$ & $1.02(0.55,1.88)$ & $1.24(0.63,2.45)$ & $1.31(0.63,2.73)$ & 0.39 \\
\hline
\end{tabular}

${ }^{a}$ Values are hazard ratios (95\% CIs) derived by Cox proportional hazards regression models adjusted for age (continuous), BMI (continuous), total energy intake (continuous), alcohol consumption (quartiles), smoking (never, past or current), energy expenditure (quartiles), social class (manual or non-manual), family history of myocardial infarction (yes or no)

${ }^{\mathrm{b}}$ Adjusted as model 1 plus sugar intake $(<50,50-100,>100 \mathrm{~g} / \mathrm{d})$, fruit consumption $(<7,8-14,15-21$, or $>21$ times/week $)$, red meat consumption $(<7,8-14,15-21$, or $>21$ times/week) and fibre (cereal and vegetable sources) $(<10,10-20$, or $>20$ g/day)

egg consumption $\geq 7$ per week compared with that of $<1$ per week over 20 years of follow-up for physicians free of previous MI. In addition, in another analysis of the same cohort there was no association between egg intake and MI and stroke, but there was a significant positive association with all-cause mortality [26]. The difference in the observed association of egg consumption and heart failure or all-cause mortality between the current study and that by Djousse et al. $[25,26]$ may be due to differences in the characteristics of the investigated subjects. Physicians were included in the study of Djousse et al. [25, 26], whereas $64 \%$ of the men in the current study were manual workers. An earlier
British study in 1997 reported a 2.68 times increased risk of ischaemic heart disease deaths in 10,802 subjects reporting higher egg intake ( $>6 /$ week) compared with lower egg consumption $(<1 \mathrm{egg} /$ week $)$ after 13.3 years follow-up [27]. In our study we were not able to conduct a similar analysis, as the data for mortality resulting from different categories of heart disease were not available.

Our finding of no association between egg intake and risk of stroke in generally healthy men is consistent with previous studies [28-30]. However, in our sub-group analysis of 340 men with T2D and/or IGT, we found a significant positive association between egg intake and the risk of stroke. 
A few epidemiological studies $[13,14]$ have investigated the association of egg consumption and CVD in T2D subjects, but none have included participants with IGT. A recent review has summarised the conflicting evidence and concluded that a diet including more eggs than is recommended (at least in some countries) may be used safely for all of the population which includes those with T2D [31]. Our study is not totally supportive of this statement and to the best of our knowledge; we are the first to show a positive association between egg consumption and stroke in subjects with T2D and/or IGT, which needs confirmation in further studies.

In our secondary cross-sectional analysis of CAPS and NDNS, a positive association between egg intake and blood glucose was observed in the T2D and/or IGT subgroup. This may indicate that higher egg intake had a detrimental effect on the glucose metabolism in subjects with T2D and/or IGT, although this needs confirmation in randomized controlled dietary intervention trials. An earlier study also showed that higher egg consumption was associated with elevated fasting glucose in 394 middle-aged healthy men [7]. This is also supported by another cross-sectional analysis [32] that observed significant positive relationships between egg consumption and fasting glucose, insulin or insulin resistance, although the difference was very small. However, neither of these studies investigated the association in sub-groups with T2D and/or IGT [7, 32].

The concept of eggs as a cholesterol rich food, which may increase LDL-cholesterol and risk of heart disease, has been recognised for a long time. However, in our analysis, we found no association between egg intake and blood cholesterol concentrations, in agreement with Gray and Griffin [10] who concluded that the effect of dietary cholesterol on LDL-cholesterol was negligible compared with the effect of dietary saturated fatty acids. In contrast to our findings, a study in a Finnish population showed a reduction in fasting plasma glucose in the highest egg consumption quartile ( $>45 \mathrm{~g} /$ day) at baseline and after a 4-year follow-up in 2312 men [12]. This significant association at baseline only appeared when dietary cholesterol intake was included as a covariate. However, controlling for dietary cholesterol may lead to bias as this component of eggs could be responsible for the observed effects of eggs on plasma glucose [33].

Lastly, this is the first study to show a strong positive association between higher egg intake and elevated $\mathrm{HbA} 1 \mathrm{c}$ concentration in a population without CVD or known T2D by using NDNS. Higher HbA1c is regarded as an important marker for pre-diagnosed T2D and CVD [34, 35]. There was no evidence in CAPS of any association between egg consumption and the development of T2D. However in a larger study, Djousse and colleagues [36] showed a significant positive association between egg consumption ( $\geq 7$ weekly) and risk of T2D in two large prospective cohort studies of men $(n=20,303)$ and women $(n=36,295)$ with 1921 and 2112 cases of T2D after a follow-up period of 20.0 years for men and 11.7 years for women, respectively. One possible explanation for the non-significant finding in CAPS is the numbers of selfreported T2D cases were relatively small and were not validated by clinical diagnosis. Nevertheless, our study agrees with the recent meta-analysis [37] which identified 12 prospective cohort studies and showed infrequent egg consumption was not associated with risk of T2D.

The potential mechanism by which eggs could increase fasting plasma glucose and ischaemic stroke in those with T2D and/or IGT is unclear. Findings from a 3-month randomized controlled study showed that there was no negative effect of higher egg consumption ( $>12$ eggs/week) on blood lipid profile compared with low egg consumption ( $<2$ eggs/week) in overweight or obese subjects diagnosed with diabetes or prediabetes [11]. However, in that study the authors controlled for diabetes or prediabetes drug use which may have masked the effect of egg consumption.

The major strength of the CAPS study is that it has a follow-up of over 20 years, which is one of the longest UK prospective cohort studies providing new evidence on the relationship between dietary factors and CVD events. Furthermore, to prevent chance findings, the cross-sectional analyses were repeated in two phases (Phase 2 and 3 ) of the CAPS (5-year interval). However, egg consumption was only recorded as the weekly egg intake and did not account for eggs consumed from composite dishes or purchased foods. Thus, egg intakes may have been underestimated or misclassified, which may have affected the observed associations. In addition, cooking methods and information on how eggs were consumed were not recorded, which may have had effects on health outcomes [38]. In terms of the metabolic markers available in CAPS, insulin was only measured in a small proportion of subjects and was not measured in Phase 3, thus insulin resistance could not be estimated.

In conclusion, our study showed no evidence for adverse effects of infrequent egg intake ( 3 eggs per week) on the risk of CVD, T2D and all-cause mortality in healthy men. However, a detrimental association of infrequent egg intake (3 eggs per week) on fasting glucose and risk of ischaemic stroke in T2D and/or IGT subjects was observed and an adverse cross-sectional association of egg consumption on $\mathrm{HbA} 1 \mathrm{C}$ in a generally healthy population. However, due to the limited sample size and number of events and deaths, cautious interpretation of these results is recommended. In addition, residual confounding factors may have influenced the association between egg consumption and health outcomes [39]. As eggs are rarely consumed alone, further research should explore the dietary pattern of high and low egg consumers, and large prospective cohort studies and RCTs are required to verify these findings in the future. 
Acknowledgements We thank Professor Yoav Ben-Shlomo, School of Social and Community Medicine, University of Bristol for managing the release of data from the CAPS.

Author contributions The authors' responsibilities were as follows: JAL and DIG designed the research; JG and DAH conducted the research; JG and DAH analysed the data with guidance from JEP; JG, DAH, DIG and JAL wrote the paper; PCE and JRC contributed expertise on epidemiology and CVD respectively. DIG and JAL had primary responsibility for the final content of the manuscript. All authors read and approved the final manuscript.

\section{Compliance with ethical standards}

Conflict of interest The authors declare that they have no conflict of interest.

Open Access This article is distributed under the terms of the Creative Commons Attribution 4.0 International License (http://creativecommons.org/licenses/by/4.0/), which permits unrestricted use, distribution, and reproduction in any medium, provided you give appropriate credit to the original author(s) and the source, provide a link to the Creative Commons license, and indicate if changes were made.

\section{References}

1. Buttar HS, Li T, Ravi N (2005) Prevention of cardiovascular diseases: role of exercise, dietary interventions, obesity and smoking cessation. Exp Clin Cardiol 10: 229-249

2. Ley SH, Hamdy O, Mohan V, Hu FB (2007) Prevention and management of type 2 diabetes: dietary components and nutritional strategies. Lancet 383:1999-2007. doi:10.1016/ S0140-6736(14)60613-9

3. Benelam B, Roe $\mathrm{M}$, Pinchen $\mathrm{H}$, Church S, Buttriss J, Gray J, Finglas P (2012) New data on the nutritional composition of UK hens' eggs. Nutr Bull 37:344-349. doi:10.1111/j.1467-3010.2012.01993.x

4. Finglas PM, Roe MA, Pinchen HM, Berry R, Church SM, Dodhia SK, Farron-Wilson M, Swan G (2014) McCance and Widdowson's the composition of foods (seventh summary edition ed.). Royal Society of Chemistry, Cambridge

5. Adamopoulos PN, Papamichael CM, Zampelas A, Moulopoulos SD (1996) Cholesterol and unsaturated fat diets influence lipid and glucose concentrations in rats. Comp Biochem Physiol B Biochem Mol Biol 113: 659 - 63

6. Lewis KE, Kirk EA, McDonald TO, Wang SR, Wight TN, O'Brien KD, Chait A (2004) Increase in serum amyloid A evoked by dietary cholesterol is associated with increased atherosclerosis in mice. Circulation 110:540-545. doi:10.1161/01. Cir.0000136819.93989.E1

7. Feskens EJ, Kromhout D (1990) Habitual dietary intake and glucose tolerance in euglycaemic men: the Zutphen Study. Int J Epidemiol 19:953-959

8. Weggemans RM, Zock PL, Katan MB (2001) Dietary cholesterol from eggs increases the ratio of total cholesterol to high-density lipoprotein cholesterol in humans: a meta-analysis. Am J Clin Nutr 73:885-891

9. Berger S, Raman G, Vishwanathan R, Jacques PF, Johnson EJ (2015) Dietary cholesterol and cardiovascular disease: a systematic review and meta-analysis. Am J Clin Nutr 102:276-294. doi:10.3945/ajen.114.100305
10. Gray J, Griffin B (2009) Eggs and dietary cholesterol-dispelling the myth. Nutr Bull 34:66-70. doi:10.1111/j.1467-3010.2008.01735.x

11. Fuller NR, Caterson ID, Sainsbury A, Denyer G, Fong M, Gerofi J, Baqleh K, Williams KH, Lau NS, Markovic TP (2015) The effect of a high-egg diet on cardiovascular risk factors in people with type 2 diabetes: the Diabetes and Egg (DIABEGG) study-a 3-mo randomized controlled trial. Am J Clin Nutr 101:705-713. doi:10.3945/ajcn.114.096925

12. Virtanen JK, Mursu J, Tuomainen TP, Virtanen HE, Voutilainen S (2015) Egg consumption and risk of incident type 2 diabetes in men: the Kuopio Ischaemic Heart Disease Risk Factor Study. Am J Clin Nutr 101:1088-1096. doi:10.3945/ajcn.114.104109

13. Rong Y, Chen L, Zhu T, Song Y, Yu M, Shan Z, Sands A, Hu FB, Liu L (2013) Egg consumption and risk of coronary heart disease and stroke: dose-response meta-analysis of prospective cohort studies. BMJ 346:e8539. doi:10.1136/bmj.e8539

14. Shin JY, Xun P, Nakamura Y, He K (2013) Egg consumption in relation to risk of cardiovascular disease and diabetes: a systematic review and meta-analysis. Am J Clin Nutr 98: 146-159. doi:10.3945/ajen.112.051318

15. Alexander DD, Miller PE, Vargas AJ, Weed DL, Cohen SS (2016) Meta-analysis of egg consumption and risk of coronary heart disease and stroke. J Am Coll Nutr 35:704-716. doi:10.1 080/07315724.2016.1152928

16. Scott J (2004) Pathophysiology and biochemistry of cardiovascular disease. Curr Opin Genet Dev 14:271-279. doi:10.1016/j. gde.2004.04.012

17. Caerphilly and Speedwell collaborative heart disease studies (1984) The Caerphilly and Speedwell collaborative group. J Epidemiol Community Health 38: 259-262

18. Fehily AM, Yarnell J, Butland BK (1987) Diet and ischaemic heart disease in the Caerphilly Study. Hum Nutr Appl Nutr 41: $319-26$

19. Bainton D, Baker IA, Sweetnam PM, Yarnell JW, Elwood PC (1988) Prevalence of ischaemic heart disease: the Caerphilly and Speedwell surveys. Br Heart J 59:201-206

20. Greenwood R, McCarron P, Elwood P, Shlomo YB, Bayer A, Baker I, Frankel S, Ebrahim S, Murray L, Smith GD (2001) The incidence and aetiology of stroke in the Caerphilly and Speedwell Collaborative Studies I: methods and incidence of events. Public Health 115:4-11. doi:10.1038/sj.ph.1900723

21. Elwood PC, Beswick A, Pickering J, McCarron P, O'Brien JR, Renaud SR, Flower RJ (2011) Platelet tests in the prediction of myocardial infarction and ischaemic stroke: evidence from the Caerphilly Prospective Study. Br J Haematol 2:514-520

22. World Health Organization (2006) Definition and Diagnosis of diabetes mellitus and intermediate hyperglycemia: report of a WHO/IDF consultation. World Health Organization, Geneva

23. Friedewald WT, Levy RI, Fredrickson DS (1972) Estimation of the concentration of low-density lipoprotein cholesterol in plasma, without use of the preparative ultracentrifuge. Clin Chem 18:499-502

24. Bates B, Lennox A, Prentice A, Bates C, Page P, Nicholson S, Swan G (2014) National Diet and Nutrition Survey: headline results from years 1 and 4 combined of the rolling programme 2008/2009-2011/12). Department of Health

25. Djousse L, Gaziano JM (2008) Egg consumption and risk of heart failure in the Physicians' Health Study. Circulation 117:512-516. doi:10.1161/CIRCULATIONAHA.107.734210

26. Djousse L, Gaziano JM (2008) Egg consumption in relation to cardiovascular disease and mortality: the Physicians' Health Study. Am J Clin Nutr 87:964-969

27. Mann JI, Appleby PN, Key TJ, Thorogood M (1997) Dietary determinants of ischaemic heart disease in health conscious individuals. Heart 78:450-455 
28. Bernstein AM, Pan A, Rexrode KM, Stampfer M, Hu FB, Mozaffarian D, Willett WC (2012) Dietary protein sources and the risk of stroke in men and women. Stroke 43:637-644. doi:10.1161/Strokeaha.111.633404

29. Larsson SC, Akesson A, Wolk A (2015) Egg consumption and risk of heart failure, myocardial infarction, and stroke: results from 2 prospective cohorts. Am J Clin Nutr 102:1007-1013. doi:10.3945/ajen.115.119263

30. Qureshi AI, Suri FK, Ahmed S, Nasar A, Divani AA, Kirmani JF (2007) Regular egg consumption does not increase the risk of stroke and cardiovascular diseases. Med Sci Monit 13:CR1-8

31. Fuller NR, Sainsbury A, Caterson ID, Markovic TP (2015) Egg consumption and human cardio-metabolic health in people with and without diabetes. Nutrients 7:7399-7420. doi:10.3390/ nu7095344

32. Djousse L, Kamineni A, Nelson TL, Carnethon M, Mozaffarian D, Siscovick D, Mukamal KJ (2010) Egg consumption and risk of type 2 diabetes in older adults. Am J Clin Nutr 92:422-427. doi:10.3945/ajcn.2010.29406

33. Kamangar F (2012) Confounding variables in epidemiologic studies: basics and beyond. Arch Iran Med 15:508-516. doi:012158/ AIM.0014
34. Peters AL, Davidson MB, Schriger DL, Hasselblad V (1996) A clinical approach for the diagnosis of diabetes mellitus: an analysis using glycosylated hemoglobin levels. Meta-analysis Research Group on the Diagnosis of Diabetes Using Glycated Hemoglobin Levels. JAMA 276:1246-1252

35. Selvin E, Steffes MW, Zhu H, Matsushita K, Wagenknecht L, Pankow J, Coresh J, Brancati FL (2010) Glycated hemoglobin, diabetes, and cardiovascular risk in nondiabetic adults. N Engl J Med 362:800-811. doi:10.1056/NEJMoa0908359

36. Djousse L, Gaziano JM, Buring JE, Lee IM (2009) Egg consumption and risk of type 2 diabetes in men and women. Diabetes Care 32:295-300. doi:10.2337/dc08-1271

37. Djousse L, Khawaja OA, Gaziano JM (2016) Egg consumption and risk of type 2 diabetes: a meta-analysis of prospective studies. Am J Clin Nutr 103:474-480. doi:10.3945/ajcn.115.119933

38. Nimalaratne C, Schieber A, Wu J (2016) Effects of storage and cooking on the antioxidant capacity of laying hen eggs. Food Chem 194:111-116. doi:10.1016/j.foodchem.2015.07.116

39. Nicklas TA, O’Neil CE, Fulgoni VL (2015) Differing statistical approaches affect the relation between egg consumption, adiposity, and cardiovascular risk factors in adults. J Nutr 145:170-176. doi:10.3945/jn.114.194068 